\title{
Element composition of additive products from electroerozive cobalt-chromum powders
}

\author{
Evgeny Ageev ${ }^{1, *}$, Alexandr Altukhov ${ }^{1}$, and Alexandr Novikov ${ }^{2}$ \\ ${ }^{1}$ South-Western State University Sciences, 305040, 50 years of October, 94, Kursk, Russia \\ ${ }^{2}$ Orel State University named after I.S. Turgenev, 302026, Komsomolskaya,. 95, Orel, Russia
}

\begin{abstract}
The wide use of the EED method for processing metal waste into powders for the purpose of their reuse and application in additive technologies is hampered by the lack in the scientific and technical literature of full-fledged information on the effect of the initial composition, regimes and media on the properties of powders and technologies of practical application. Therefore, in order to develop technologies for the reuse of electroerosive powders and to evaluate the effectiveness of their use, complex theoretical and experimental studies are required. The aim of the work was to conduct X-ray spectral microanalysis of additive products from electroerosive cobalt-chrome powders. For the implementation of the planned studies, wastes of the cobaltochrome alloy of the brand KHMS "CELLIT" were chosen. As a working fluid, butyl alcohol (butanol-1) was used. For the production of cobalt-chrome powders, a unit for EED of conductive materials was used. Dispersion parameters: voltage $100 \mathrm{~V}$, capacity $48 \mu \mathrm{F}$, repetition rate $120 \mathrm{~Hz}$. The microstructure of the samples (on the surface) was studied by scanning electron microscopy. The surface of the samples was ground and polished. With the help of an energy-dispersive X-ray analyzer from EDAX, built into the scanning electron microscope QUANTA 200 3D, spectra of characteristic X-ray radiation were obtained at various points on the surface of the sample and along the cross-section. Based on the results of $\mathrm{X}$-ray spectral analysis of additive products from electroerosive cobaltchrome powders, it has been established experimentally that the main elements in them are $\mathrm{Co}$, Mo, and $\mathrm{Cr}$.
\end{abstract}

\section{Introduction}

The technology of "three-dimensional printing" appeared in the late 80-ies of the last century. The pioneer in this field is the company 3D Systems, which developed the first commercial stereolithographic machine - SLA - Stereolithography Apparatus (1986).

The widespread use of digital technologies in the field of CAD, modeling and calculation (CAE) and machining (CAM) has stimulated the explosive nature of the development of printing technologies, and now it is extremely difficult to indicate the area of material production where to some extent printers would not have been used.

\footnotetext{
*Corresponding author: ageev_ev@mail.ru
} 
Additive technologies (AT) production of products from materials based on metals and alloys is one of the most promising and actively developing areas of production.

The main requirement for powders for additive $3 \mathrm{~d}$ technologies is the spherical shape of the particles. Such particles are most compactly packed into a certain volume and ensure the "fluidity" of the powder composition in the supply systems of the material with minimal resistance. In addition, the powder should contain a minimum amount of dissolved gas. The microstructure of the powder must be uniform and finely dispersed (with a uniform distribution of phase constituents) [1-8].

Proceeding from the peculiarities of the methods for obtaining spherical powders with the aim of obtaining spherical granules of regulated granularity, the electroerosive dispersion technology is proposed, which is characterized by relatively low energy costs and ecological purity of the process [9-12].

The main advantage of the proposed technology is the use of waste as raw materials, which is much cheaper than the pure components used in traditional technologies. In addition, this technology is powdered, which allows powder-alloys.

The wide use of the EED method for processing metal waste into powders for the purpose of their reuse and application in additive technologies is hampered by the lack in the scientific and technical literature of full-fledged information on the effect of the initial composition, regimes and media on the properties of powders and technologies of practical application. Therefore, in order to develop technologies for the reuse of electroerosive powders and to evaluate the effectiveness of their use, complex theoretical and experimental studies are required.

The aim of the work was to study the elemental composition of additive products from electroerosive cobalt-chrome powders.

\section{Materials and methods}

For the implementation of the planned studies, wastes of the cobaltochrome alloy of the brand KHMS "CELLIT" were chosen. As a working fluid, butyl alcohol (butanol-1) was used. For the production of cobalt-chrome powders, a unit for EED of conductive materials was used. Dispersion parameters: voltage $100 \mathrm{~V}$, capacity $48 \mu \mathrm{F}$, repetition rate $120 \mathrm{~Hz}$.

The microstructure of the samples (on the surface) was studied by scanning electron microscopy. The surface of the samples was ground and polished. Grinding was made with metallographic paper with a large (No. 60-70) and fine grain (№oo 220-240). During the grinding process, the sample was periodically rotated by $90^{\circ}$. The abrasive particles were washed with water and subjected to polishing on a circle with suspensions of metal oxides $\left(\mathrm{Fe}_{3} \mathrm{O}_{4}, \mathrm{Cr}_{2} \mathrm{O}_{3}, \mathrm{Al}_{2} \mathrm{O}_{3}\right)$. After achieving a mirror gloss, the surface of the section was washed with water, alcohol and dried with filter paper. With the help of an energydispersive X-ray analyzer from EDAX, built into the scanning electron microscope QUANTA 200 3D, spectra of characteristic X-ray radiation were obtained at various points on the surface of the sample and along the cross-section. By X-ray spectral microanalysis is meant the determination of the elemental composition of microobjects by the characteristic $\mathrm{X}$-ray radiation excited in them. For the analysis of the characteristic spectrum in X-ray spectral analysis, two types of spectrometers are used (uncrystalline or with a crystal analyzer), the base for X-ray spectral analysis is the electron-optical system of a scanning electron microscope. When the electronic probe interacts with the sample (Fig.1 and 2), one of the excited signals is X-ray radiation, which can be divided into: characteristic and inhibitory. Braking X-ray radiation arises from the deceleration of primary electrons in the electric (Coulomb) field of the atoms of the material being analyzed. The kinetic energy of primary electrons in this case is partially or completely transformed into energy of X-ray 
radiation. Accordingly, the radiation has a continuous spectrum with energy from zero to the energy of the incident electron and therefore it is also called continuous X-ray radiation. With x-ray spectral microanalysis, bremsstrahlung is undesirable, since it makes the main contribution to increasing the background level and can not be ruled out. When primary electrons penetrate the sample, they are inhibited not only by the electric field of the atoms, but also by a direct collision with the electrons of the atoms of the material. As a result, primary electrons can knock out electrons from internal K-, L- or M-shells, leaving the sample atom in the energetically excited state. The forming vacancies are filled with electron transitions from higher energy levels. The atom passes to the ground state, the excess energy is released as an X-ray quantum. Since the energy of the emerging quantum depends only on the energy of the electronic levels participating in the process, and they are characteristic for each element, characteristic X-ray radiation arises. So each atom has a definite finite number of levels, between which only certain types of transitions are possible, the characteristic X-ray emission gives a discrete line spectrum.

X-ray spectral microanalysis can not determine light elements with an atomic number less than 4 in the alloy. There are such difficulties with the identification of elements when lines of the L- or M-series of another element are superimposed on the line of the K-series of one element. An important characteristic of the X-ray spectral analysis is its locality, i.e. the volume of the substance in which the characteristic X-ray radiation is excited. It is determined primarily by the diameter of the electronic probe on the sample and depends on the accelerating voltage and the chemical composition of the material (Fig. 2).

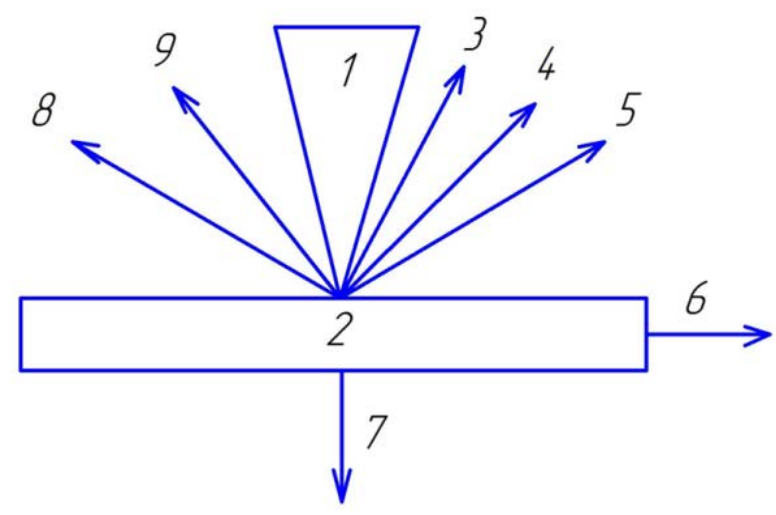

Fig. 1. Effects of the interaction of an electron beam with an object. 1 - electron beam, 2- object, 3 - reflected electrons, 4 - secondary electrons, 5 - Auger electrons, 6 - current of absorbed electrons, 7 - transmitted electrons, 8 - cathode-luminescence radiation, 9 - x-ray emission 


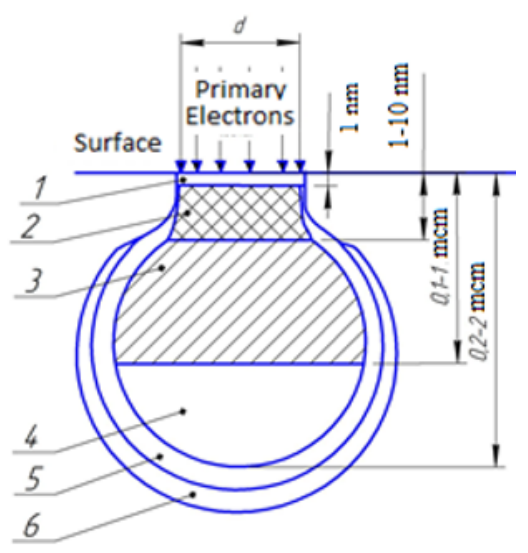

Fig. 2. Signal domains and spatial resolution when the surface of an object is irradiated with an electron beam (probe). Areas of generation: 1 - Auger electrons, 2 - secondary electrons, 3 reflected electrons, 4 - characteristic X-ray radiation, 5-brake X-rays, 6 - fluorescence

Analysis of the distribution of elements can be performed in qualitative, semiquantitative and quantitative form. Qualitative analysis determines the type of elements included in the composition of the sample section of the sample. If the sample has several phases (sections) whose chemical composition is unknown, then a qualitative analysis of each phase is performed. Qualitative analysis is usually used to determine the nature of the distribution of elements over the area of the section. After the qualitative analysis, the quantitative analysis is often carried out at separately selected points, according to the obtained data, the software allows to determine the phase type, based on its chemical composition. Semiquantitative analysis is realized if it is required to determine the distribution of elements along the lines (linear analysis). Linear analysis is performed using the step-scan method, i.e. by sequential analysis at individual points. Thus, a quantitative determination of the concentration of elements with a given accuracy is carried out. The spectra of characteristic X-ray radiation correspond to the points in the figure. On the spectrum, each chemical element corresponds to a peak of a certain height. Detecting reflected electrons. Some models of microscopes are equipped with a highly sensitive semiconductor detector for back-scattered electrons. The detector is mounted on the lower surface of the objective lens or is inserted on a special rod under the pole piece. This allows, by selecting a mode from the menu, to obtain images of the topography of the surface, an image in compositional contrast or in a dark field. When an electron beam hits the sample, some electrons can escape from the surface of the sample as a result of their interaction with the crystal lattice of the sample. On the fluorescent screen, back scattered electrons create a picture called the backscattering pattern of electrons or the Kikuchi lines. The diffraction of backscattered electrons makes it possible to obtain information about the texture and orientation of grains of crystalline samples, to map the orientations of the crystal lattice (i.e., the distribution of orientations over the sample). The diffraction of backscattered electrons also makes it possible to analyze the defects of the microstructure, allows the analysis of the phases from which a solid is composed, separates the grains and determines their boundaries, analyzes the homogeneity of the material, and analyzes microdeformations and microstrains. If necessary, such an analysis is possible in comparison with the images in the secondary and reflected electrons, in the characteristic $\mathrm{X}$-ray emission (of the interesting elements of the composition) of the same sections of the sample. 


\section{Results and discussion}

The results of microscopy and microanalysis of the samples are shown in Fig. 3 and 4.

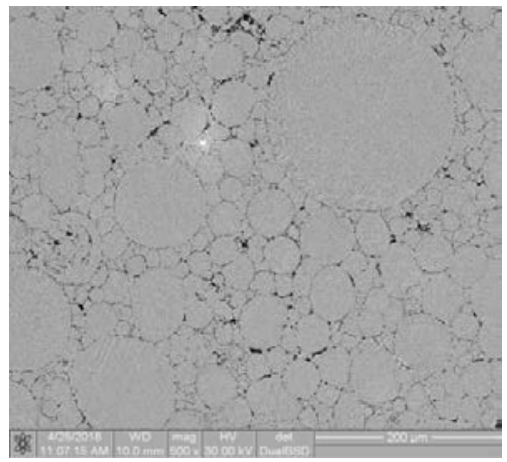

a

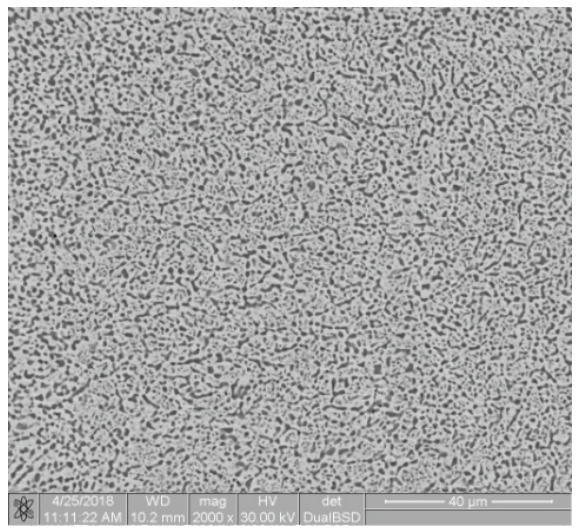

c

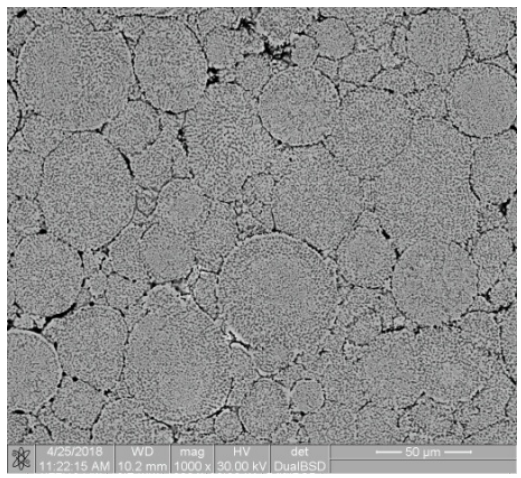

b

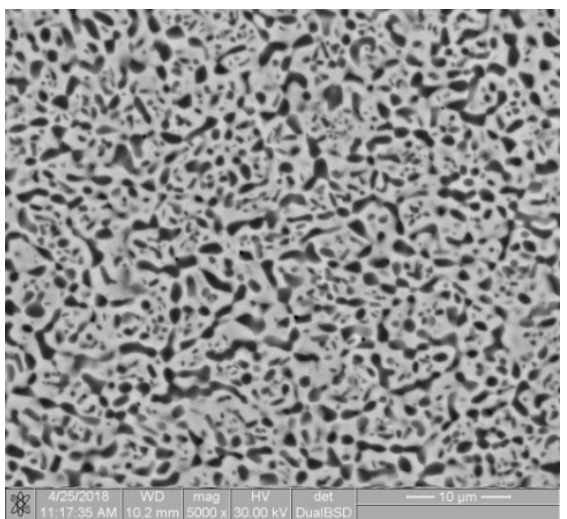

d

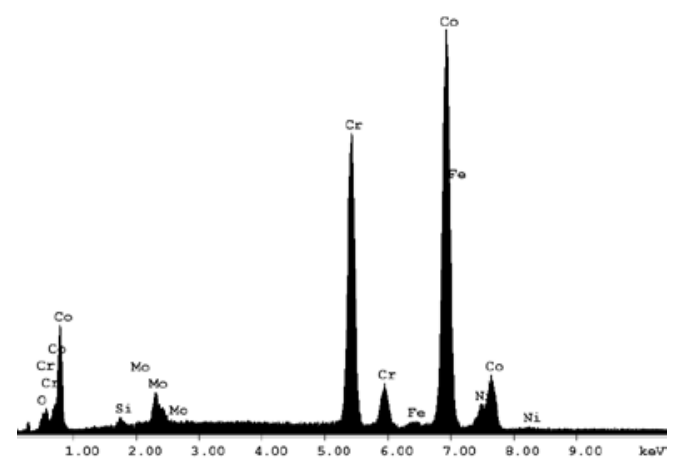

e

Fig. 3. Microstructure (Quanta 200 3D, BSE - backscattered electron mode): a) x500; (b) x1000; (c) x2000; (d) x5000; e) elemental composition of the sample surface 


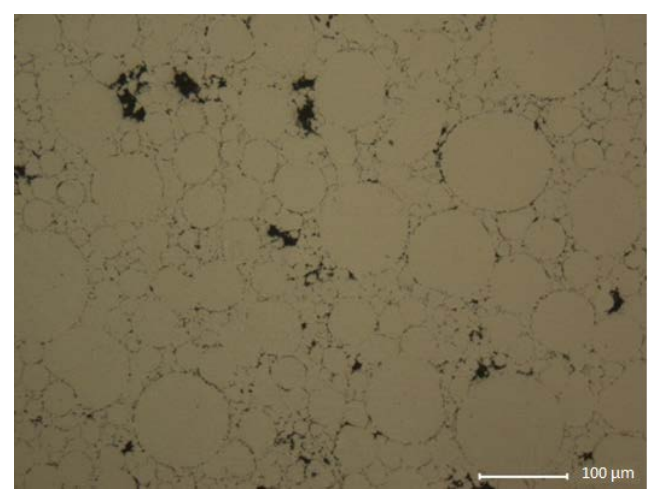

a

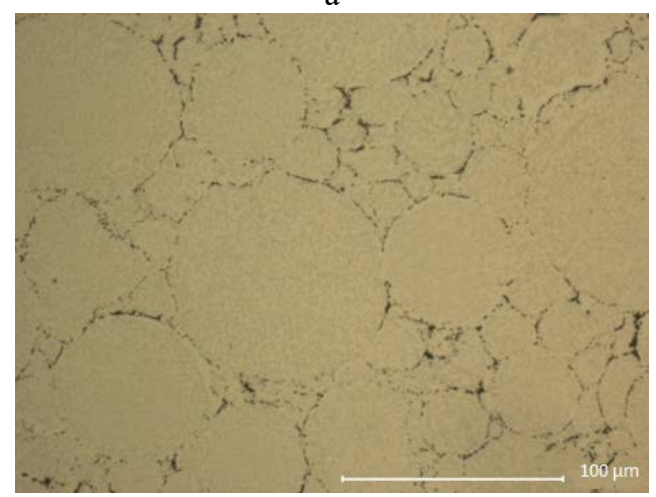

b

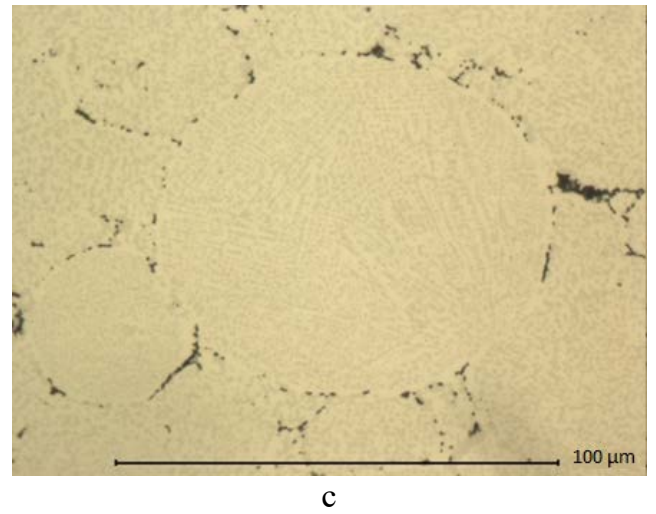

Fig. 4. Microstructure (optical microscope): a) x200; b) x500; c) x1000

\section{Conclusion}

Based on the results of X-ray spectral analysis of additive products from electroerosive cobalt-chrome powders, it has been established experimentally that the main elements in them are $\mathrm{Co}, \mathrm{Mo}$, and $\mathrm{Cr}$.

The work was supported by the Russian Science Foundation. Project number is 17-79-20336. 


\section{References}

1. Loeber L., Biamino S., Ackelid U. et al. Comparison of Selective Laser and Electron Beam Melted Titanium Aluminides. Conference paper of 22nd International symposium "Solid freeform fabrication proceedings", University of Texas, Austin, 2011, pp. 547556.

2. Song B., Dong S., Zhang B. et al. Effects of processing parameters on microstructure and mechanical property of selective laser melted Ti6Al4V. Materials \& Design, Vol. 35, pp. 120-125 (2012).

3. Gu D.D., Meiners W., Wissenbach K., Poprawe R. Laser additive manufacturing of metallic components: materials, processes and mechanisms. International Materials Reviews, Vol. 57 (3), pp. 133-164 (2012)

4. Biamino S., Penna A., Ackelid U et al. Electron beam melting of Ti-48Al-2Cr-2Nb alloy: microstructure and mechanical properties investigation. Intermetallics, Vol. 19, pp. 776-781 (2011).

5. Song B., Dong S., Coddet P. et al. Fabrication and microstructure characterization of selective laser melted FeAl intermetallic parts. Surface and Coatings Technology, Vol. 206, pp. 4704-4709 (2012)

6. Safdar A., He H.Z., Wei L.Y., Snis A. et al. Effect of process parameters settings and thickness on surface roughness of EBM produced Ti-6Al-4V, Rapid Prototyping Journal, Vol. 18 (5), pp.401-408 (2012).

7. Wang Z., Guana K., Gaoa M. The microstructure and mechanical properties of deposited-IN718 by selective laser melting. Journal of Alloys and Compounds, Vol. 513, pp. 518-523 (2012).

8. Safdar A., Wei L.Y., Snis A., Lai Z. Evaluation of microstructural developmentin electron beam melted Ti-6Al-4V. Materials Characterization, Vol. 65, pp. 8-15 (2012).

9. E.V. Ageeva, A.Yu. Altukhov, S.V. Khardikov, S.S. Gulidin, A.N. Novikov, Electroerosive Powder Obtained from Alloy VK8 Waste into Butanol, Journal of nanoand electronic physics, Vol. 7, No 4, Part 2, P. 04080(3) (2015).

10. E.V. Ageeva, E.V. Ageev, S.V. Pikalov, E.A. Vorobiev, A.N. Novikov, X-ray Analisis of the Powder of Micro- and Nanometer Fractions, Obtained from Wastes of Alloy T15K6 in Aqueous Medium, Journal of nano- and electronic physics, Vol. 7, No 4, Part 2, P. 04058(2) (2015).

11. .V. Ageeva, E.V. Ageev, V.Yu. Karpenko, A.S. Osminina, Studing Tungstencontaining Electroerosion Powders and Alloys Synthesized from Them, Journal of nano- and electronic physics, Vol. 3, P. 03049-1-03049-3 (2014).

12. E.V. Ageeva, E.V. Ageev, N.M. Horyakova, V.S. Malukhov, Production of copper electroerosion nanopowders from wastes in kerosene medium, Journal of nano- and electronic physics, Vol. 3, P. 03011-1 - 03011-3 (2014). 